\title{
Exact Signal Model and New Carrier Frequency Offset Compensation Scheme for OFDM
}

\author{
Baile Xie, Student Member, IEEE, Wenxun Qiu, Student Member, IEEE, and Hlaing Minn, Senior Member, IEEE
}

\begin{abstract}
Carrier frequency offset (CFO) causes spectrum misalignment of transmitter and receiver filters. This misalignment leads to energy loss and distortion of received signal, resulting in performance degradation of the whole system. However, these issues are often overlooked by existing works. This paper presents an exact signal model for OFDM that takes into account the CFO-induced spectral misalignment and related aliasing effects. Incorporating these practical issues, we also propose a new preamble structure and a new CFO compensation approach to mitigate their negative effects. Theoretical analysis and simulation results substantiate the importance of the exact signal model and the advantage of the proposed CFO compensation scheme.
\end{abstract}

Index Terms-Carrier frequency offset, CFO compensation, OFDM, preamble design, signal model, spectrum misalignment.

\section{INTRODUCTION}

$\mathbf{O}$ RTHOGONAL frequency-division multiplexing (OFDM) is vulnerable to the carrier frequency offset (CFO) arising from oscillator inaccuracies. The CFO introduces inter-carrier interference (ICI), which may cause serious performance degradation [1], [2]. The demands for low-cost devices, semiconductor downscaling trend, support of higher mobile speeds [3], and the use of higher frequency bands make the CFO compensation task more important for future wireless systems.

Practical systems use a transmitter (Tx) pulse shaping filter for spectral emission control and a receiver (Rx) filter for a matched reception. With $\mathrm{CFO}$, the transmitted spectrum becomes misaligned with the bandwidth of the Rx filter, inducing signal energy loss and distortion. Most of the previous works related to OFDM with CFO (e.g., [4]-[8]) overlooked this practical effect. The existing signal model is actually an approximation which holds only for small CFO. The spectrum misalignment was incorporated in [9] for deriving CramerRao bound (CRB) of CFO estimation for a single carrier system in additive white Gaussian noise (AWGN) channel, and in [10], [11] for optimizing the pulse shaping filters for OFDM in AWGN channels. Recently, it is included in [12], [13] in frequency-selective channels but it still involves an approximation by regarding the convolution of sampled individual signal components as directly sampling the overall signal. It is crucial to have an accurate signal model since it not only provides technical accuracy and reference to approximate

Manuscript received August 7, 2011; accepted November 1, 2011. The associate editor coordinating the review of this letter and approving it for publication was X. Wang.

The authors are with the Department of Electrical Engineering, University of Texas at Dallas, Richardson, TX, 75080, USA (e-mail: \{baile.xie, wxq081000, hlaing.minn\}@utdallas.edu).

Digital Object Identifier 10.1109/TWC.2011.120611.111490 models, but may also reveal new characteristics which might have been missed in approximate models.

In this paper, we propose an exact signal model that incorporates the CFO-induced spectral misalignment and related aliasing effects for OFDM systems in frequency-selective fading channels. Several aspects that the existing signal model fails to capture are analyzed, followed by new insights and their impacts on system design and bit error rate (BER) performance in OFDM and orthogonal frequency-division multiple access (OFDMA) systems. By capitalizing these knowledge from the exact signal model, a new preamble structure and a new CFO compensation scheme are proposed which provide better BER performance than the existing scheme.

The rest of the paper is organized as follows. Section II briefly describes the signal model used in existing literature. The exact signal model is introduced and then compared with the existing one in Section III. In Section IV, we propose a new preamble structure and a new CFO compensation approach. Simulation results are presented in Section V and conclusion is drawn in Section VI.

\section{Existing Signal Model for OFDM with CFO}

Consider an OFDM system consisting of $N$ subcarriers with cyclic prefix (CP) length $N_{\mathrm{cp}}$. The discrete-time sample index set corresponding to the $p$ th OFDM symbol is denoted by $J_{p}=\left\{p N^{\prime}, p N^{\prime}+1, \ldots,(p+1) N^{\prime}-1\right\}$, with $N^{\prime}=N+N_{\mathrm{cp}}$. Consider a time-invariant (within an OFDM symbol) multipath fading channel having $L$ paths with path gains $\left\{h_{k}: k=\right.$ $0,1, \ldots, L-1\}$ and path delays $\left\{\tau_{k}: k=0,1, \ldots, L-1\right\} . H_{n}$ denotes the corresponding channel gain for the $n$th subcarrier. After the CP removal at the Rx, the $m$ th sample of the $p$ th OFDM symbol is denoted as $r_{p}[m] \triangleq r\left(t_{p}[m]\right)$, for $m=$ $0, \ldots, N-1$, and $t_{p}[m] \triangleq\left(p N^{\prime}+N_{\mathrm{cp}}+m\right) T+t_{0}$, where $t_{0}$ is a delay related to Rx filter output sampling and $1 / T$ is the sampling frequency ( $N$ times the subcarrier spacing).

In the existing signal model, the received signal in discretetime domain with perfect timing is given by [4]

$r_{p}[m]=\frac{1}{\sqrt{N}} e^{j \theta} e^{j 2 \pi t_{p}[m] \Delta_{f}} \sum_{n=0}^{N-1} a_{p, n} H_{n} e^{j \frac{2 \pi}{N} m n}+w\left(t_{p}[m]\right)$

where $\theta$ is an arbitrary phase shift and $\Delta_{f}$ is the CFO in Hz. The sequence $\left\{a_{p, n}, 0 \leqslant n \leqslant N-1\right\}$ represents the independent zero-mean transmitted data (or contains pilots randomly selected from a predefined set of pilots). $\left\{w\left(t_{p}[m]\right)\right\}$ are the AWGN samples. The frequency domain signal on the 
$l$ th subcarrier of the $p$ th OFDM symbol is

$$
\begin{aligned}
R_{p, l}= & \frac{Q_{p}}{N} \sum_{n=0}^{N-1} a_{p, n} H_{n} e^{j \frac{\pi}{N}(N-1)(n-l+v)} \frac{\sin \pi(n-l+v)}{\sin \frac{\pi}{N}(n-l+v)} \\
& +W_{p, l}
\end{aligned}
$$

where $v \triangleq N \Delta_{f} T$ is the CFO normalized by the subcarrier spacing, $W_{p, l}$ is the frequency-domain noise sample, and $Q_{p} \triangleq e^{j\left(2 \pi \Delta_{f} t_{p}[0]+\theta\right)}$. The signal to interference ratio (SIR) on the $l$ th subcarrier of the $p$ th OFDM symbol is given by

$$
\operatorname{SIR}_{p, l}=\frac{E\left[\left|a_{p, l}\right|^{2}\right] E\left[\left|H_{l}\right|^{2}\right] \frac{\sin ^{2}(\pi v)}{\sin ^{2}\left(\frac{\pi}{N} v\right)}}{\sum_{n=0, n \neq l}^{N-1} E\left[\left|a_{p, n}\right|^{2}\right] E\left[\left|H_{n}\right|^{2}\right] \frac{\sin ^{2} \pi(n-l+v)}{\sin ^{2} \frac{\pi}{N}(n-l+v)}} .
$$

For a channel with uncorrelated channel paths, we have $E\left[\left|H_{n}\right|^{2}\right]=\sum_{k=0}^{L-1} E\left[\left|h_{k}\right|^{2}\right]$, which is the same across the subcarriers. Given an equal average energy of transmitted data $E\left[\left|a_{p, n}\right|^{2}\right]$ for $n=0, \cdots, N-1$, SIR can be simplified as

$$
\mathrm{SIR}_{l}=\frac{\frac{\sin ^{2}(\pi v)}{\sin ^{2}\left(\frac{\pi}{N} v\right)}}{\sum_{n=0, n \neq l}^{N-1} \frac{\sin ^{2} \pi(n-l+v)}{\sin ^{2} \frac{\pi}{N}(n-l+v)}} .
$$

Note that in this case the SIR (a function of $v$ only) is essentially the same across subcarriers. The average energy of time-domain noise-free sample from (1) (denoted by $\tilde{r}_{p}[m]$ ) is

$$
E\left[\left|\tilde{r}_{p}[m]\right|^{2}\right]=\frac{1}{N} \sum_{n=0}^{N-1} E\left[\left|a_{p, n}\right|^{2}\right] E\left[\left|H_{n}\right|^{2}\right]
$$

which is independent of CFO in the existing signal model.

\section{EXact Signal Model for OFDM with CFO}

In this section, we develop an exact signal model for OFDM in frequency-selective fading channels to capture the spectral misalignment effects of $\mathrm{Tx}$ and $\mathrm{Rx}$ filters in the presence of CFO. For mathematical preciseness, we begin with continuous-time signals. The transmitted signal is given as $s(t)=\sum_{k} s_{k} g(t-k T)$, where $s_{k}$ is the discrete-time transmitted signal and $g(t)$ is the Tx pulse shaping filter (or Tx-side equivalent filter). The impulse response of a multipath fading channel is $h(t)=\sum_{i=0}^{L-1} h_{i} \delta\left(t-\tau_{i}\right)$. Let $g_{R}(t)$ represent the $\mathrm{Rx}$ filter impulse response and $z(t)$ denote the $\mathrm{Rx}$ filter output noise. Then, the received signal $r(t)$ is written as

$$
\begin{aligned}
r(t) & =\left[\sum_{k} s_{k} \sum_{i=0}^{L-1} h_{i} g\left(t-\tau_{i}-k T\right) e^{j 2 \pi \Delta_{f} t} e^{j \theta}\right] * g_{R}(t)+z(t) \\
& =e^{j \theta} e^{j 2 \pi \Delta_{f} t} \sum_{k} s_{k} \sum_{i=0}^{L-1} x_{i}\left(t-k T, \Delta_{f}\right)+z(t) \\
& =e^{j \theta} e^{j 2 \pi \Delta_{f} t} \sum_{k} s_{k} x\left(t-k T, \Delta_{f}\right)+z(t)
\end{aligned}
$$

where $^{1}$

$$
\begin{aligned}
& x_{i}\left(t-k T, \Delta_{f}\right)=\left[h_{i} g\left(t-\tau_{i}-k T\right)\right] *\left[g_{R}(t) e^{-j 2 \pi \Delta_{f} t}\right], \\
& \sum_{i=0}^{L-1} x_{i}\left(t-k T, \Delta_{f}\right) \\
& =\sum_{i=0}^{L-1} \int h_{i} g\left(t-\tau-\tau_{i}-k T\right) e^{-j 2 \pi \Delta_{f} \tau} g_{R}(\tau) d \tau \\
& \stackrel{(a)}{=} \int H(f) G(f) G_{R}\left(f+\Delta_{f}\right) e^{j 2 \pi f(t-k T)} d f \\
& \triangleq x\left(t-k T, \Delta_{f}\right)
\end{aligned}
$$

with $H(f), G(f)$ and $G_{R}(f)$ representing the Fourier transform of $h(t), g(t)$ and $g_{R}(t)$, respectively. The step $(a)$ holds by invoking Fourier transform.

Consider $s_{k}=s_{p, \tilde{k}}=s_{p N^{\prime}+N_{\mathrm{cp}}+\tilde{k}}$ as the $\tilde{k}$ th timedomain transmit sample of the $p$ th OFDM symbol, where $\tilde{k} \triangleq\left(k\right.$ modulo $\left.N^{\prime}\right)-N_{\text {cp }}$. After sampling the Rx filter output signal $r(t)$ in (6), the $m$ th sample of the $p$ th received OFDM symbol reads as

$$
\begin{aligned}
r_{p}[m]= & Q_{p} e^{j 2 \pi \Delta_{f} m T} \sum_{\tilde{k}=-N_{\mathrm{cp}}}^{N-1} s_{p, \tilde{k}} x\left(t_{0}+m T-\tilde{k} T, \Delta_{f}\right) \\
& +z\left(t_{p}[m]\right),
\end{aligned}
$$

with $m=0, \ldots, N-1$ after $\mathrm{CP}$ removal. The $l$ th received tone of the $p$ th OFDM symbol is

$$
\begin{aligned}
R_{p, l}= & \frac{Q_{p}}{\sqrt{N}} \sum_{m=0}^{N-1} \sum_{\tilde{k}=-N_{\mathrm{cp}}}^{N-1} s_{p, \tilde{k}} x\left(t_{0}+m T-\tilde{k} T, \Delta_{f}\right) \\
& \times e^{j 2 \pi m\left(\Delta_{f} T-\frac{l}{N}\right)}+Z_{p, l}
\end{aligned}
$$

where $Z_{p, l}$ is the noise term. In practice, $x\left(t, \Delta_{f}\right)$ in (8) has a finite support. Suppose $t_{0}=K_{1} T$, and the discrete-time version of the equivalent channel $x\left(t, \Delta_{f}\right)$ has $K_{1}$ taps at the left of the sampling instant $t_{0}$ and $K_{2}-1$ taps at the right of $t_{0}$. $\sum_{\tilde{k}=-N_{\mathrm{cp}}}^{N-1} s_{p, \tilde{k}} x\left(t_{0}+m T-\tilde{k} T, \Delta_{f}\right)$ is equivalent to the shifted version of circular convolution of $\left\{s_{p, 0}, s_{p, 1}, \cdots, s_{p, N-1}\right\}$ and $\left\{x\left(0, \Delta_{f}\right), x\left(T, \Delta_{f}\right), \cdots, x\left(\left(K_{1}+K_{2}-1\right) T, \Delta_{f}\right)\right\}$. Alternatively, we can resort to the DFTs of the two. Note that $s_{p, \tilde{k}}=\frac{1}{\sqrt{N}} \sum_{n=0}^{N-1} a_{p, n} e^{j 2 \pi n \tilde{k} / N}$ and

$$
X\left[l, \Delta_{f}\right] \triangleq \sum_{m=0}^{K_{1}+K_{2}-1} x\left(m T, \Delta_{f}\right) e^{-j 2 \pi m l / N}
$$

is the DFT of $x\left(m T, \Delta_{f}\right)$. Further manipulation of (10) yields

$$
\begin{aligned}
R_{p, l}= & \frac{Q_{p}}{N} \sum_{n=0}^{N-1} a_{p, n} e^{j 2 \pi K_{1} n / N} X\left[n, \Delta_{f}\right] e^{j \frac{\pi}{N}(N-1)(n-l+v)} \\
& \times \frac{\sin \pi(n-l+v)}{\sin \frac{\pi}{N}(n-l+v)}+Z_{p, l} .
\end{aligned}
$$

${ }^{1}$ The signals on the right side of (7) are practically time-limited, and hence they are not band-limited. The discrete-time version of each individual signal is essentially an aliased version of the original signal. Thus sampling individual components and convolving the resulting discrete-time signals (as done in [12] [13]) will not equal the discrete-time signal obtained by sampling the signal on the left hand side. In our derivation, we still keep analyzing in the continuous-time domain at this point. 
Comparing (12) and (2), one can observe that besides $e^{j 2 \pi K_{1} n / N}, H_{n}$ in (2) of the existing signal model is substituted by $X\left[n, \Delta_{f}\right]$, which, from (8) and (11), can also be expressed as

$$
X\left[n, \Delta_{f}\right]=\left.\frac{1}{T} \sum_{k=-\infty}^{+\infty} \tilde{X}\left(f-\frac{k}{T}\right)\right|_{f=\frac{n}{N T}},
$$

where

$$
\tilde{X}(f)=G(f) H(f) G_{R}\left(f+\Delta_{f}\right)
$$

is the frequency response of the continuous-time equivalent channel: a concatenation of the Tx filter, the channel and the misaligned Rx filter. Equation (13) represents the discrete version of (14) after both time- and spectral-domain sampling. Intuitively, in the presence of CFO (and hence the spectral misalignment), the frequency response of $G(f) G_{R}\left(f+\Delta_{f}\right)$ no longer remains flat after sampling. Therefore, rather than simply transforming the channel impulse response $h(t)$ into $H_{n}$ by DFT as in the existing signal model, $X\left[n, \Delta_{f}\right]$ captures the CFO-induced spectral misalignment and the Tx and Rx filtering as well as related aliasing effects. Thus, $X\left[n, \Delta_{f}\right]$ offers a more comprehensive perspective on the actual transmission system, serving as a benchmark in assessing the approximation made by the existing signal model.

As a result, the SIR on the $l$ th subcarrier of the $p$ th OFDM symbol is

$$
\operatorname{SIR}_{p, l}=\frac{E\left[\left|a_{p, l}\right|^{2}\right] E\left[\left|X\left[l, \Delta_{f}\right]\right|^{2}\right] \frac{\sin ^{2}(\pi v)}{\sin ^{2}\left(\frac{\pi}{N} v\right)}}{\sum_{n=0, n \neq l}^{N-1} E\left[\left|a_{p, n}\right|^{2}\right] E\left[\left|X\left[n, \Delta_{f}\right]\right|^{2}\right] \frac{\sin ^{2} \pi(n-l+v)}{\sin ^{2} \frac{\pi}{N}(n-l+v)}} .
$$

Provided the same average transmit data energy over all subcarriers, (15) simplifies to

$$
\operatorname{SIR}_{l}=\frac{E\left[\left|X\left[l, \Delta_{f}\right]\right|^{2}\right] \frac{\sin ^{2}(\pi v)}{\sin ^{2}\left(\frac{\pi}{N} v\right)}}{\sum_{n=0, n \neq l}^{N-1} E\left[\left|X\left[n, \Delta_{f}\right]\right|^{2}\right] \frac{\sin ^{2} \pi(n-l+v)}{\sin ^{2} \frac{\pi}{N}(n-l+v)}} .
$$

Note that the general SIR expressions (15) and (3) are of similar form except the difference of $X\left[l, \Delta_{f}\right]$ versus $H_{l}$, but (16) and (4) differ more in that the terms $E\left[\left|X\left[n, \Delta_{f}\right]\right|^{2}\right]$ cannot be canceled out in (16) since the CFO-induced misalignment and related aliasing effect render the equivalent channel power gain $E\left[\left|X\left[n, \Delta_{f}\right]\right|^{2}\right]$ vary from subcarrier to subcarrier even for uncorrelated channel paths.

Averaging the energy of the received discrete-time signal sample without noise, we get

$$
E\left[\left|\tilde{r}_{p}[m]\right|^{2}\right]=\frac{1}{N} \sum_{n=0}^{N-1} E\left[\left|a_{p, n}\right|^{2}\right] E\left[\left|X\left[n, \Delta_{f}\right]\right|^{2}\right] .
$$

It is clear that the received signal energy is a function of CFO whereas it is not in the existing signal model as shown in (5).

With (12)-(14), we can easily visualize how the CFO affects the equivalent channel energy in the frequency domain: the tones shifted outside the Rx filter spectrum are attenuated, causing substantial energy loss on those subcarriers, which is further complicated by the aliasing effect due to spectral replication as shown in (13). The whole spectrum of the

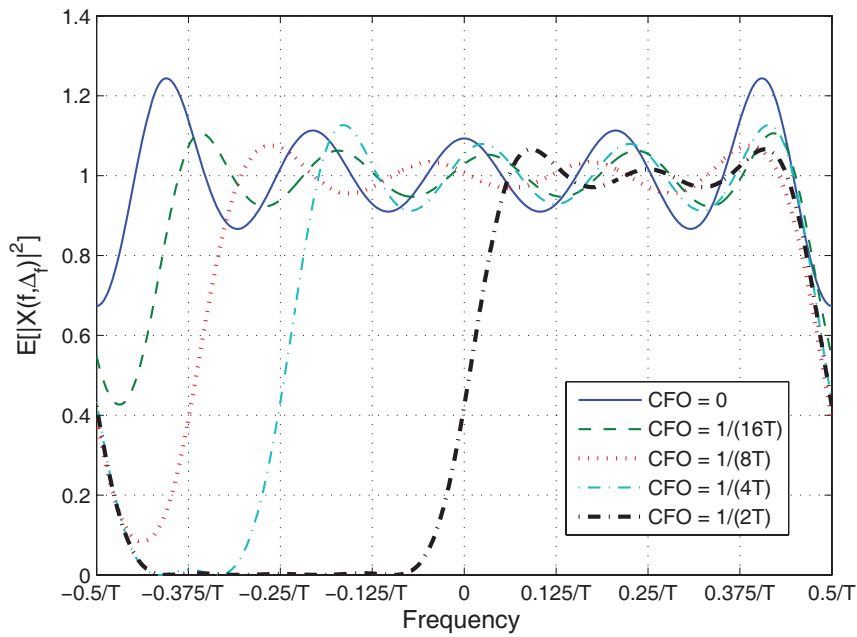

Fig. 1. Average equivalent channel power gain $E\left[\left|X\left[f, \Delta_{f}\right]\right|^{2}\right]$ in the presence of CFO $(g(t)$ is with the support $[-5 T, 5 T]$ and $\beta=0.12)$

received signal is no longer flat and the larger the CFO is, the less the received signal energy remains. The above effects are not reflected in the existing signal model which always yields optimistic received signal energy without any energy loss.

To get some intuition, let us consider a numerical example where $g(t)$ and $g_{R}(t)$ are both square root raised cosine (SRRC) with support $-5 T \leqslant t \leqslant 5 T$ and roll-off factor $\beta=0.12$. Without loss of generality, we set $E\left[\left|a_{p, n}\right|^{2}\right]=1$, $E\left[\left|H_{n}\right|^{2}\right]=1$ and $N=64$. To obtain a broad picture of how the equivalent channel power gain $E\left[\left|X\left[f, \Delta_{f}\right]\right|^{2}\right]$ corresponds to different $\mathrm{CFO}$ values, we show a large CFO range from 0 to $1 /(2 T)$ in Fig. 1. It is observed that as the CFO increases, the portion of the band suffering energy suppression grows. A limiting case would be only half of the spectrum remains when $\mathrm{CFO}=1 /(2 T)$. In addition, the average energy of the entire time-domain received signal is plotted in Fig. 2. Compared with a constant signal energy level of the existing model regardless of the CFO, the one in the exact model decreases approximately in proportion to the amount of CFO with respect to the entire band. One might attempt to adjust the existing signal model to account for the signal energy loss by multiplying the received signal with a scaler dependent on CFO. However, this method changes the energy on every subcarrier uniformly, and hence fails to capture uneven error performances among subcarriers. Therefore, this remedy is not appealing, especially for OFDMA systems.

In general, for large $\mathrm{CFO}$ values, the signal energy loss and distortion revealed by the exact signal model become significant that they undermine the reliability of data transmission (especially for the subcarriers around the band edge). Therefore, efficient CFO compensation schemes which could tackle the problem are desirable.

\section{Preamble structure and CFO Compensation}

The current trend of CFO compensation uses the digital compensation approach (i.e., multiplying the baseband received samples with CFO-compensating phasors) to remove the frequency offset of the received signal. The frame structure used in the existing approach consists of, as shown in 


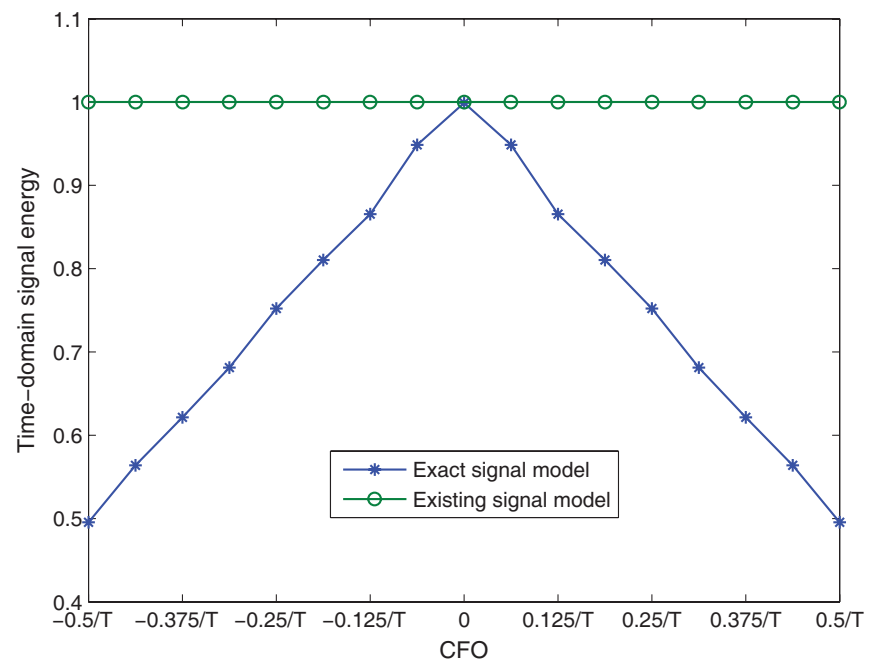

Fig. 2. Average total energy of time-domain received signal versus $\mathrm{CFO}$ in an OFDM system

Fig. 3 (a), preambles for synchronization (PS), preambles for channel estimation (PC), and data. Nevertheless, the phase counter-rotation cannot help recover the lost signal energy since the energy loss has already occurred after the signal passes through the misaligned Rx filter.

To overcome the above issue, we propose a new approach which adopts a new frame structure and introduce an analog CFO compensation, which refers to some procedure performed before the signal is converted to digital domain (i.e., adjusting oscillator frequency which is used to mix with the received radio frequency analog signal). Specifically, in our proposed approach, according to CFO estimation based on the PS of current frame, an adjustment of the local oscillator frequency is performed. Practically, once the PS reaches at the Rx, the CFO can be estimated ${ }^{2}$ while subsequent transmitted signals keep arriving. Modified from the existing preamble structure, a null duration is inserted between the PS and PC in the new preamble format, as depicted in Fig. 3 (b). The null duration buys the $\mathrm{Rx}$ the time for $\mathrm{CFO}$ estimation and oscillator frequency adjustment. Consequently, the ensuing Rx output signal incurs a CFO as small as the estimation error, resulting in only negligible energy loss.

A case in point is as follows. Consider the PS and PC are of the same length, which are evenly divided into 10 blocks respectively. All blocks share the same signal energy. Periodic training sequence is used for both $\mathrm{CFO}$ and channel estimations. Since the training sequences are periodic and known at the Rx, only one block is used as CP in the existing approach for each preamble as in Fig. 3 (c). Note that with a proper sequence design, the CFO estimation performance mainly depends on the preamble length and energy [14] while the channel estimation mainly depends on the preamble energy [15], [16] but not on the preamble length provided that the length is longer than or equal to the equivalent channel length.

\footnotetext{
${ }^{2}$ The energy loss and distortion experienced by the received PS affect the accuracy of CFO estimation, which in turn depends on the value of CFO, the PS and filter designs. The accuracy of CFO estimation can be assessed by deriving CRB based on the exact signal model, which however is out of the scope of this paper.
}

Hence, it is recommended that the null portion be created mostly from the PC while almost allocating the same amount of energy for channel estimation. In the proposed approach, assuming that the $\mathrm{CFO}$ estimation and oscillator adjustment can be done in one block duration, we keep PS the same, reducing one block from the existing $\mathrm{PC}$, namely, 9 blocks for the new PC as illustrated in Fig. 3 (d). ${ }^{3}$ After CP removal, there are both 9 blocks for $\mathrm{CFO}$ estimation and channel estimation in the existing approach, compared to 9 blocks for CFO estimation and 8 blocks for channel estimation in the proposed approach, respectively. As a result, the preamble length and energy of the proposed approach stay the same as the existing approach for CFO estimation, whereas its useful channel estimation preamble energy is $80 / 81$ of that of the existing approach. The performances of the proposed compensation scheme compared to the existing one will be evaluated by simulation in next section.

\section{Simulation Results}

In the simulation, we assume a 4-tap Rayleigh fading channel with an exponential power delay profile $(3 \mathrm{~dB}$ per tap decaying factor). The modulation scheme is 16 QAM and DFT size is 128. For the sake of computational efficiency, we use the ad hoc estimator (essentially best linear unbiased estimator) from [8]. A periodic training sequence of period of 4 samples for CFO estimation is used and it follows the preamble structures described in Section IV, in particular, Fig. 3 (c) and (d) with a CP length of 32 . For coded cases, we apply the industrial standard convolutional code with rate $1 / 2$, constraint length 7 , generator matrix $[133,177]$ and the Viterbi decoding. The same parameter settings of $g(t)$ and $g_{R}(t)$ are used as in Section III.

Let us consider a typical oscillator instability of $\pm 10 \mathrm{ppm}$. For communication over $60 \mathrm{GHz}$ band, one of the emerging areas, this oscillator instability translates into CFO of \pm 600 $\mathrm{kHz}$, which is $\pm 0.06 / T$ for a sampling frequency (approximately the bandwidth) of $10 \mathrm{MHz}$. In this case, the CFO could be amount to $0.12 / T$. In the following, we mainly use two CFO values: $0.06 / T$ and $0.12 / T$ for illustration. Note that CFO could be much larger under certain conditions/scenarios. For example, the ambient temperature of the device could be well outside the typical range for which the oscillator accuracy is guaranteed. The low cost implementation as well as aging might deteriorate the oscillator accuracy. High Doppler shift (as experienced in highway, train, bullet train, etc.) will also increase the CFO.

Fig. 4 shows the uncoded BER per tone of the two approaches at $E_{b} / N_{0}=13 \mathrm{~dB}$ and $\mathrm{CFO}=0.06 / T$, where $E_{b}$ is the bit energy and $N_{0}$ is the noise power spectral density. It is clear that for the existing approach, subcarriers on the bandedge (corresponding to the middle tones in Fig. 4) experience higher error probability than its counterparts in the proposed approach. The proposed approach amends the energy loss by adjusting the oscillator frequency based on the CFO

\footnotetext{
${ }^{3}$ To keep the same energy as the existing PC, each block of the new PC is of energy 10/9 times that of the existing PC. If the time for CFO estimation and oscillator adjustment is less than one block, the null duration can be reduced accordingly and the block energy of the new preamble can be accordingly adjusted.
} 


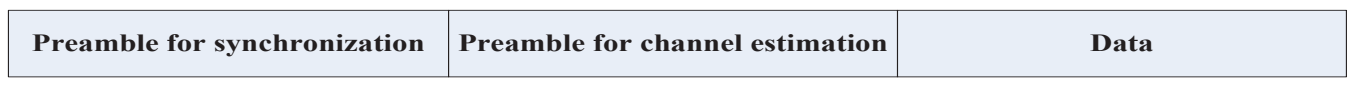

(a) Existing frame structure

\begin{tabular}{|c|c|c|c|}
\hline Preamble for synchronization & Null & $\begin{array}{c}\text { Preamble for channel } \\
\text { estimation }\end{array}$ & Data \\
\hline
\end{tabular}

(b) Proposed frame structure

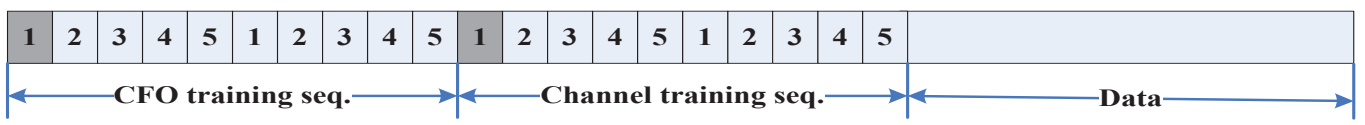

(c) Example of existing frame structure

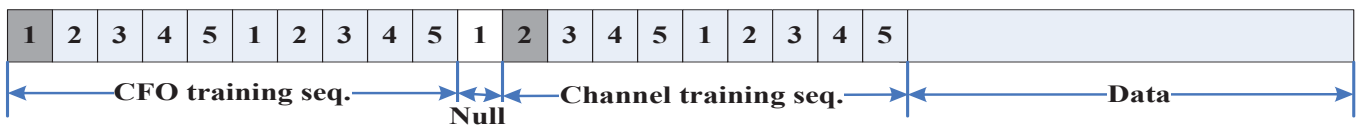

(d) Example of proposed frame structure

Fig. 3. Frame structures of the existing and new approaches for CFO compensation

estimation. For the proposed approach, the SNR on the data symbols corresponds to the scenario with virtually no CFO, provided an accurate CFO estimation is in place.

Since the BER varies across tones under the exact signal model, we can expect an uneven BER performance for different users in an OFDMA system. We simulate a simplified OFDMA system with 128 subcarriers and 8 users who evenly share the whole bandwidth with a localized resource allocation (i.e., the subcarriers assigned to a user is contiguous). Fig. 5 shows the average coded BER performances of two users A and $\mathrm{B}$, who occupy the subcarriers numbered from 56 to 71 , and 72 to 87 , respectively. For User B, the two approaches perform approximately the same. However, User A, which is around the band edge, suffers about $1.5 \mathrm{~dB}$ SNR loss if it uses the existing compensation approach compared with employing the proposed approach.

The overall BER performance in an OFDM system with CFO of $0.06 / T$ and $0.12 / T$ are presented in Fig. 6. Both coded and uncoded cases demonstrate that the proposed approach outperforms the existing one and the advantages of the proposed approach is more noticeable under coding. For instance, when $\mathrm{CFO}=0.12 / T$, about $1 \mathrm{~dB}$ SNR advantage is achieved by the new compensation approach with coding. The results further legitimate the necessity of the exact signal model as well as the new compensation approach when the $\mathrm{CFO}$ is large.

Note that our simulations and discussions assume that all subcarriers are utilized for transmission. In several current OFDM-based wireless communication standards, a certain number of null or virtual tones are placed on band edges, which are originally designed mainly for transmit spectrum control and for the ease of filter design. With the exact signal model, we find that these null tones could also help reduce the energy loss on the band edge, at the cost of spectral efficiency. However, in the presence of such null tones, the pilot design for channel estimation may not be mean-square-error-optimal [15]. On the other hand, the use

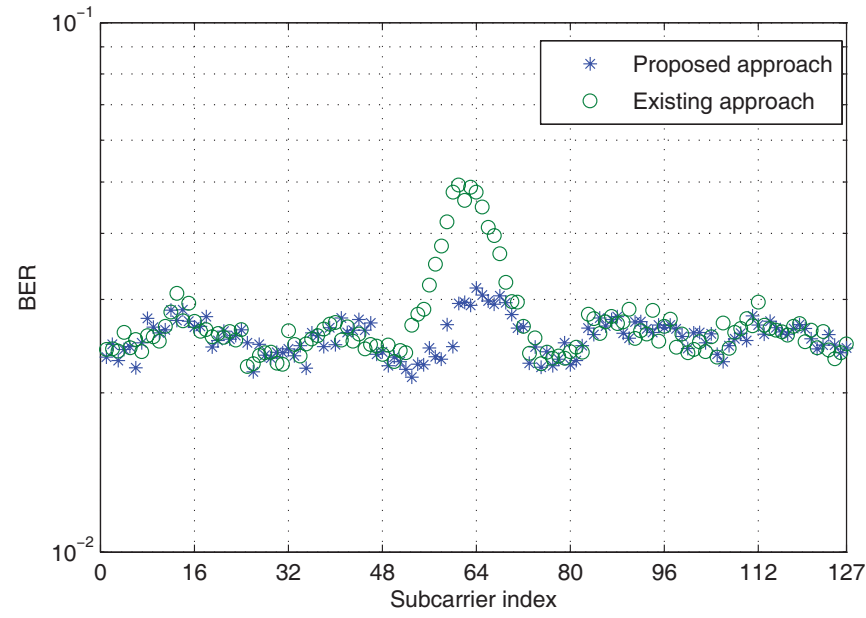

Fig. 4. BER across subcarriers for two CFO compensation approaches for uncoded case, where $\mathrm{CFO}=0.06 / T, N=128$, and $E_{b} / N_{0}=13 \mathrm{~dB}$ (subcarrier index here corresponds to DFT index)

of virtual subcarriers is just one of the approaches to satisfy the transmission spectral mask. With an appropriate filter design and subcarrier spacing (for example, using filter with small roll-off factor and/or reducing the subcarrier spacing), all subcarriers can be used for transmission while meeting the transmission mask. Therefore, we do not limit our signal model to a specific practical implementation, but we aim at a general system where null subcarriers may or may not be used. More importantly, for future systems which use less or no null tones, the characteristics revealed by our signal model need to be properly incorporated in the system design. The proposed compensation approach serves as a promising solution since it does not impose any requirement on null tones.

\section{CONCLUSIONS}

This paper proposes an exact signal model for OFDM systems with CFO in frequency-selective fading channels. 


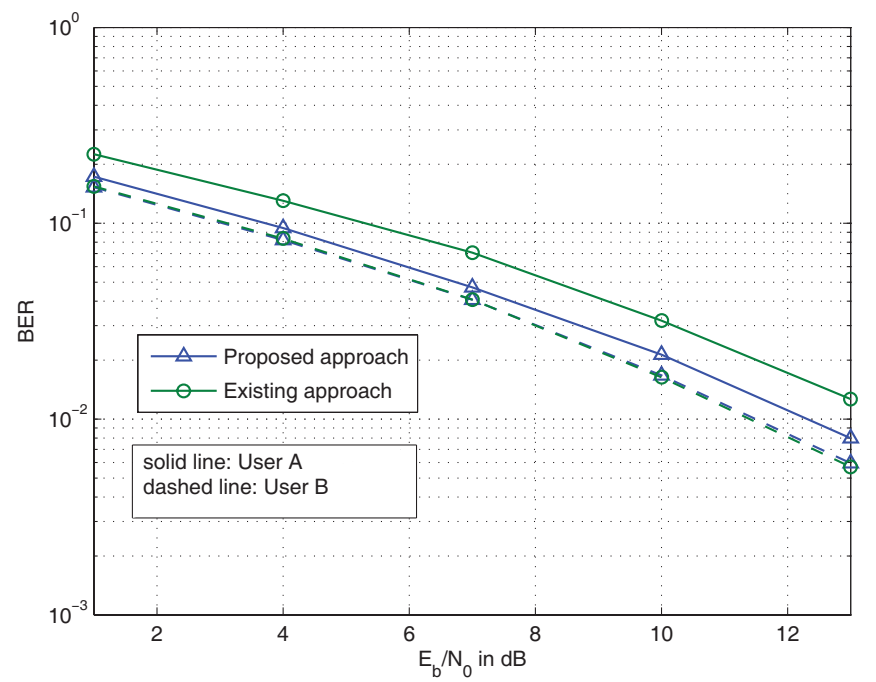

Fig. 5. Coded BER versus SNR per bit for two CFO compensation approaches in an OFDMA system with $N=128$ and CFO $=0.06 / T$

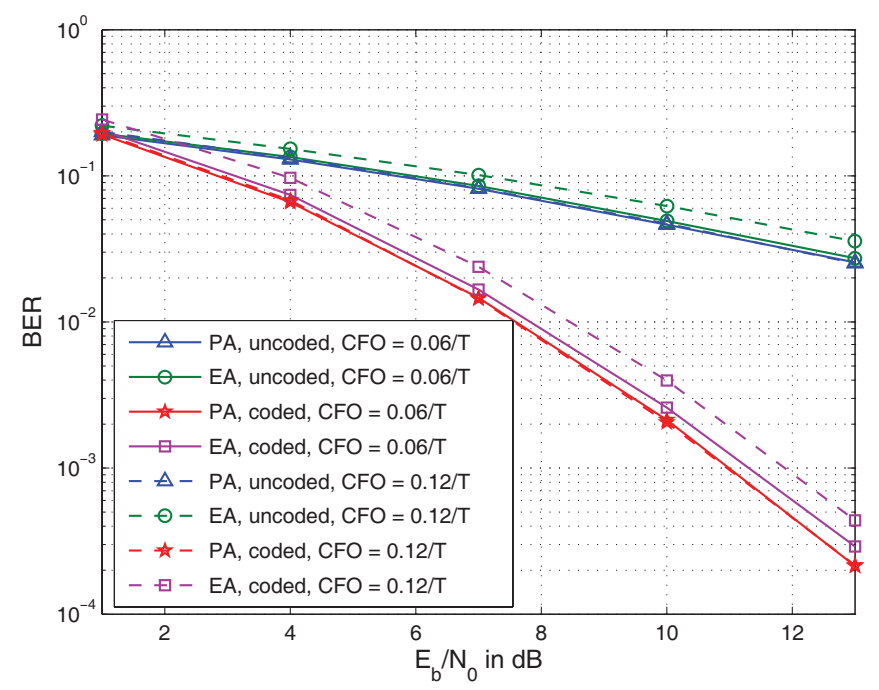

Fig. 6. BER versus SNR per bit for two CFO compensation approaches in an OFDM system with $N=128$, PA: Proposed Approach, EA: Existing Approach

It is revealed that the CFO-induced spectral misalignment between the $\mathrm{Tx}$ and $\mathrm{Rx}$ filters as well as related aliasing effect lead to the energy loss and distortion of the received signal, which causes further performance degradation in data detection. The subcarriers around the band edge suffer more and hence transmitting signaling or control information on these subcarriers is not advisable. In addition, resource al- location in OFDMA systems should appropriately take into account this effect. Given the new effects revealed by the exact signal model, a new preamble structure and a new CFO compensation approach are proposed, which effectively solve these issues. Simulation results confirm advantages of the proposed approach over the existing one.

\section{REFERENCES}

[1] L. J. Cimini, "Analysis and simulation of a digital mobile channel using orthogonal frequency division multiplexing," IEEE Trans. Commun., vol. 33, pp. 665-675, July 1985.

[2] T. Pollet, M. V. Bladel, and M. Moeneclaey, "BER sensitivity of OFDM systems to carrier frequency offset and Wiener phase noise," IEEE Trans. Commun., vol. 43, pp. 191-193, Feb./Mar./Apr. 1995.

[3] WP5D, "Draft report on requirements related to technical performance for IMT-Advanced radio interface(s) [IMT.TECH]," Working Party 5D Sub-Working Group Radio Aspects, 2nd Meeting, Dubai, United Arab Emirates, Jul. 2008.

[4] P. H. Moose, "A technique for orthogonal frequency division multiplexing frequency offset correction," IEEE Trans. Commun., vol. 42, no. 10, pp. 2908-2914, Oct. 1994.

[5] J. van de Beek, M. Sandell, and P. Bjorjesson, "ML estimation of time and frequency offset in OFDM systems," IEEE Trans. Signal Processing, vol. 45, pp. 1800-1805, July 1997.

[6] M. Speth, S. A. Fechtel, G. Fock, and H. Meyr, "Optimum receiver design for wireless broad-band systems using OFDM-part I," IEEE Trans. Commun., vol. 47, pp. 1668-1677, Nov. 1999.

[7] M. Luise and R. Reggiannini, "Carrier frequency acquisition and tracking for OFDM systems," IEEE Trans. Commun., vol. 44, pp. 1590-1598, Nov. 1996.

[8] M. Morelli and U. Mengalli, "Carrier-frequency estimation for transmissions over selective channels," IEEE Trans. Commun., vol. 48, pp. 1580-1589, Sep. 2000.

[9] N. Noels, H. Steendam, and M. Moeneclaey, "The true Cramer-Rao bound for carrier frequency estimation from a PSK signal," IEEE Trans. Commun., vol. 52, pp. 834-844, May 2004.

[10] P. Tan and N. C. Beaulieu, "Reduced ICI in OFDM systems using the "better than" raised cosine pulse," IEEE Commun. Lett., vol. 12, no. 3, pp.135-137, Mar. 2004.

[11] G. Lin, L. Lundheim, and N. Holte, "Optimal pulses robust to carrier frequency offset for OFDM/QAM systems," IEEE Commun. Lett., vol. 8, pp. 161-163, Mar. 2008.

[12] G. Xing, M. Shen, and H. Liu, "Frequency offset and I/Q imbalance compensation for direct-conversion receivers," IEEE Trans. Wireless Commun., vol. 4, pp. 673-680, Mar. 2005.

[13] H. Lin and K. Yamashita, "Subcarrier allocation based compensation for carrier frequency offset and I/Q imbalances in OFDM systems," IEEE Trans. Wireless Commun., vol. 8, no. 1, pp. 18-23, Jan. 2009.

[14] H. Minn, X. Fu, and V. K. Bhargava, "Optimal periodic training signal for frequency offset estimation in frequency-selective fading channels," IEEE Trans. Commun., vol. 54, no. 6, pp. 1081-1096, June 2006.

[15] H. Minn and N. Al-Dhahir, "Optimal Training Signals for MIMO OFDM Channel Estimation," IEEE Trans. Commun., vol. 5, no. 5, pp. 11581168, May 2006.

[16] H. Minn, N. Al-Dhahir, and Y. Li, "Optimal training signals for MIMO OFDM channel estimation in the presence of frequency offsets and phase noise," IEEE Trans. Commun., vol. 54, no. 10, pp. 1754-1759, Oct. 2006. 